A Social Theory of Corruption 



\title{
A Social Theory of Corruption
}

\author{
NOTES FROM THE
}

INDIAN SUBCONTINENT

\section{Sudhir Chella Rajan}


Copyright $\odot 2020$ by the President and Fellows of Harvard College All rights reserved

Printed in the United States of America

FIRST PRINTING

Cover design: Annamarie McMahon Why

Cover art: Aleksandr Zubkov @ Getty Images

9780674250406 (EPUB)

9780674250413 (MOBI)

9780674250420 (PDF)

The Library of Congress has cataloged the printed edition as follows:

Names: Rajan, Sudhir Chella, 1961- author.

Title: A social theory of corruption : notes from the Indian subcontinent /

Sudhir Chella Rajan.

Description: Cambridge, Massachusetts : Harvard University Press, 2020.

Includes bilbiographical references and index.

Identifiers: LCCN 2020018588 | ISBN 9780674241275 (cloth)

Subjects: LCSH: Corruption-India-History. | Dominance (Psychology)—India-

History. | Elite (Social sciences) -India-History. | India-Social conditions.

Classification: LCC HV6771.I4 R35 2020 | DDC 364.1/3230954-dc23

LC record available at https://lccn.loc.gov/2020018588 
In memory of my father, Sunder Rajan, whose learning was graced with patience and humility 
